Special issue of the 3rd International Conference on Computational and Experimental Science and Engineering (ICCESEN 2016)

\title{
A Cyclic Voltammetry Study on Electrodeposition of Cu-Zn Alloy Films: Effect of Ultrasonication Time
}

\author{
K. YURDAL* AND İ.H. KaRAHAN \\ Mustafa Kemal University, Physics Department, Hatay, Turkey
}

\begin{abstract}
Copper zinc alloys were electrodeposited in the form of thin films. Electrodeposition process was carried out in non-cyanide aqueous medium, under constant current. Effect of pre-deposition ultrasonication of solution was investigated. In other words ultrasonic treatment was applied only before the electrodeposition. Solutions were subjected to high-energy ultrasonication for increasing times. Cyclic voltammetry was used to understand changes occurring due to duration of ultrasonication. No ultrasonication was applied during deposition. Atomic composition of resultant films were revealed by MP-AES. It was found that implementation of ultrasonic treatment before electrodeposition affects CV characteristics, when compared to non-ultrasonicated solution. Atomic composition of fabricated samples were found to be close to each other, some of them showing more deviation.
\end{abstract}

DOI: 10.12693/APhysPolA.132.1087

PACS/topics: 81.05.Bx, 68.35.bd

\section{Introduction}

Metallic thin films are scientifically important materials and find many technologic applications in industry. Therefore fabrication and characterization of these materials are extensively studied. CuZn alloys have many applications both in bulk and thin film forms. These applications include protection against corrosion, use as substrate material for film deposition, adhesive interlayers, uses due to good mechanical properties and machinability, decorative purposes etc. [1-9]. In addition, copper and some of its other alloys are important materials for research [10-12].

Another important property of CuZn alloy group is shape memory effect. NiTi alloys are another important group of shape memory materials [13]. Shape memory effect can be described as material ability to change its shape upon heating. Shape change may be preserved upon cooling, which is called one way shape memory effect, or material can exist in different shapes at different temperature intervals and can switch between these different shapes, which is called two way shape memory effect [14].

Shape change during transformation is accompanied by changes in physical and chemical properties. Therefore, shape memory materials can be used as actuators and sensors [15]. When shape memory materials are fabricated in the form of thin films, they can be used as micro-valves, micro grippers, micro actuators etc. They can be integrated into silicon technology. Furthermore they have a potential to be used in MEMS.

For thin films to be utilized in industry, it is important to be able to fabricate materials with required physical and chemical properties, which is only possible

\footnotetext{
*corresponding author; e-mail: kaganyurdal@hotmail.com
}

through theoretical and experimental research. There are many thin and thick film production techniques, each with its own advantages and drawbacks [16-20]. Among these techniques, electrodeposition has advantages of non-vacuum fabrication, ability of production at room temperature, aqueous deposition, low cost, versatility etc.

Non-cynide solutions are preferred as deposition medium because of environmental and health concerns. Some researchers reported studies on electrodeposition of CuZn alloys [21, 22] and some others underlined shape memory property $[23,24]$. Many researchers used ultrasound during electrodeposition of metallic materials and metal matrix composites. Tudela et al. studied ultrasound assisted electrodeposition of nickel. They reported that presence of ultrasound caused a preferred orientation.

Grain refinement, together with work-hardening resulted in harder Ni films [25]. Kim et al. used ultrasonication in electrodeposition of $\mathrm{Cu} / \mathrm{Al}_{2} \mathrm{O}_{3}$ nanocomposite thin films. They reported that ultrasound provides reduced agglomeration of nanoparticles and resulted in a finer grained $\mathrm{Cu}$ matrix, resulting in better mechanical properties [26].

In this study we research the effect of ultrasonication time on solution properties and atomic composition of resultant films. Ultrasonication was applied before deposition. In other words, electrodeposition was carried out in non-ultrasonicated conditions. Ultrasonication is used to generate cavitation in solution and change solution characteristics by applying high energy. It was considered that type and/or amount of formed complexes may be changed by this energy input, which will result in different deposition characteristics.

\section{Experimental}

Electrodeposition was carried out using a PARSTAT 2273 galvanostat/potentiostat, using an aqueous 
medium, at room temperature. Aluminum plates, with dimensions of $2 \times 2 \mathrm{~cm}^{2}$, were used as substrates. Substrates were cleaned and activated by dipping into $1 \mathrm{M}$ $\mathrm{NaOH}$ solution and rinsing with distilled water. A platinum wire was used as counter electrode. Experimental grade chemicals were used.

$0.06 \mathrm{M} \mathrm{CuSO}_{4} \cdot 5 \mathrm{H}_{2} \mathrm{O}$ and $0.1 \mathrm{M} \mathrm{ZnSO}_{4} \cdot 7 \mathrm{H}_{2} \mathrm{O}$ were used as metallic ion sources. $0.5 \mathrm{M}$ trisodium citrate dihydrate (TSC Dihydrate) was used as complexing agent, to form metallic complexes and to provide stability for deposition.

Electrodeposition was carried out galvanostatically at current density of $15 \mathrm{~mA} / \mathrm{cm}^{2}$. Solutions were subjected to ultrasonication before electrodeposition for 5-60 minutes. A Hielscher UP 200S type ultrasonicator was used at a cycle time of 0.5. Ultrasonication was achieved by directly dipping the ultrasound probe into the solution. A non-ultrasonicated solution was used as reference for comparison. Solution behaviors were examined using cyclic voltammetry between $0.6 \mathrm{~V}$ and $-1.8 \mathrm{~V}$ potentials. Films were peeled off from substrates and dissolved in acidic medium to reveal atomic composition using MPAES. Solutions and samples are named according to ultrasonication period. For example U5 is the solution and respective sample for 5 minutes of ultrasonication.

\section{Results and discussion}

Cyclic voltammetry (CV) is a widely used and powerful tool in electrochemistry, which reveals solution behavior and makes it possible to predict ideal conditions for electrodeposition. Figure 1 shows CV graphs of four samples which were selected as representative samples for $\mathrm{CV}$, which gives a comparison for non-ultrasonicated sample (U0) and samples with minimum and maximum ultrasonication (U5 and U60), as well as average sample with 30 minutes of ultrasonication (U30). U30 sample has curled and separated from substrate during deposition, which has probably affected film properties.

This figure makes it possible to understand the overall effect of ultrasonication, covering a wide range of ultrasonication periods. It is observed that shape of $\mathrm{CV}$ graph for reference solution (U0), which was not subjected to ultrasonication, shows a relatively different behavior. Cathodic current, which indicates deposition of metal ions onto the substrate, starts at more negative potentials, compared to ultrasonicated samples.

Moreover cathodic current at $-1.8 \mathrm{~V}$ maximum potential is lower than that of ultrasonicated solutions. Also, shape of nose at maximum negative potential is narrower than in other samples. Maximum cathodic current for non-ultrasonicated sample is around $-26 \mathrm{~mA}$, while 5,30 , and 60 minute ultrasonicated samples all have a $35 \mathrm{~mA}$ current at $-1.8 \mathrm{~V}$ potential.

Upon reversal of the potential towards the positive values, two peaks are observed at around $-0.5 \mathrm{~V}$ and $0.1 \mathrm{~V}$. These two peaks are called anodic peaks and they refer to peeling of the formed film layer. Two distinct peaks refer to separate dissolution of $\mathrm{Cu}$ and $\mathrm{Zn}$ elements due to their different reduction potentials. U0 sample shows relatively low current values upon dissolution as well, which is in compliance with lower cathodic current. Ultrasonicated samples show very similar behaviors both in cathodic and anodic current regions.

Figure 2 gives another comparison of ultrasonicated samples for more detailed understanding of changes. Figure 2 consists of the data obtained from Fig. 1. That is to say, ultrasonication period has made only small changes in CV graphs.

Figure 3 shows potential vs. elapsed time for nonultrasonicated sample as well for samples ultrasonicated during 5, 30 and 60 minutes. It is observed that when solution is subjected to ultrasonication before electrodeposition, there is a tendency for deposition at less negative potential values. This means that forming the same current in solution needs less driving force, in other words species in the solution are a bit more active than in non-ultrasonicated ones. This effect is most visible in 30 minute ultrasonicated solution.

However, this tendency does not increase as time increases further, since 60 minute ultrasonicated solution has shown a more negative potential profile, which is close to that of non-ultrasonicated sample.

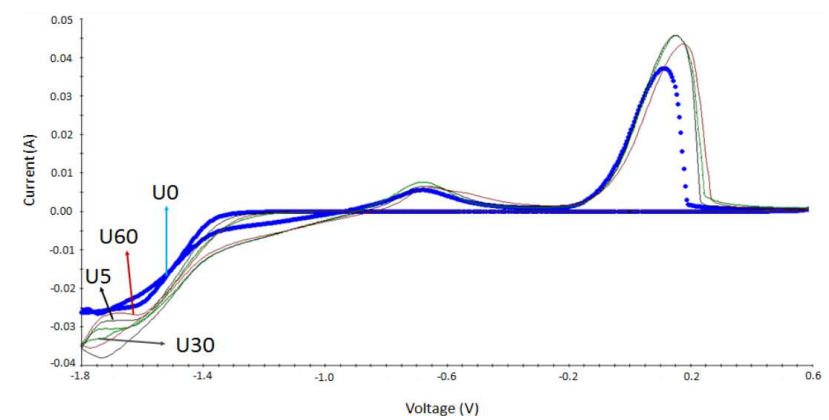

Fig. 1. Comparative CV graphs of samples with zero, maximum, minimum and half-maximum ultrasonication.

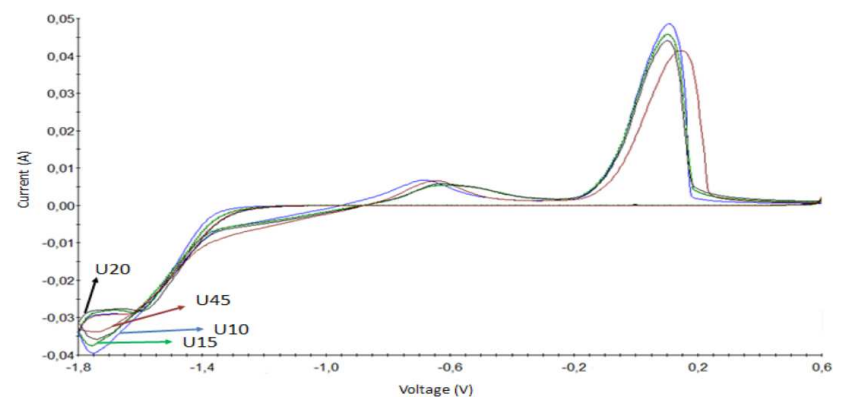

Fig. 2. Comparative CV graphs of 10, 15, 20 and 45 minute ultrasonicated solutions.

Difference in behaviors of cyclic voltammetry and potential vs time graphs may be attributed to type and/or 


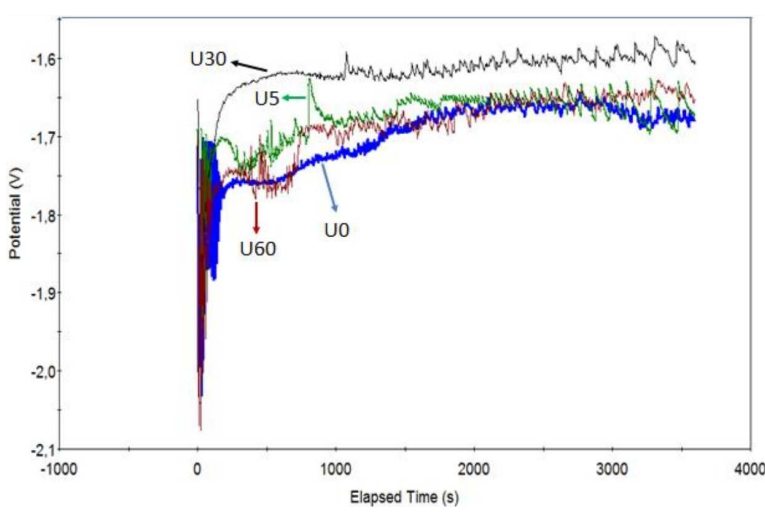

Fig. 3. Potential vs. elapsed time graphs for electrodeposition from non-ultrasonicated (U0), 5, 30, and 60 minutes ultrasonicated (U5, U30 and U60, respectively) solutions.

amount of formed complexes in the solution, or some other changes, which affect mobility of charged species. This difference may be due to various reasons, such as acidity, temperature, concentration of ions etc. In this case it is attributed to ultrasonic treatment.

High energy applied by ultrasonication causes some changes in solution equilibrium. However it is seen that ultrasonication period does not have significant effect on shape of CV graph.

Some researches have focused on examining the types and amount of complexes formed in $\mathrm{Cu}$ and $\mathrm{Zn}$ solutions in which tri sodium citrate is used as the complexing agent. Sangmin Shin et al. reported a study focusing on reduction potentials of $\mathrm{Cu}$ and $\mathrm{Zn}$, with and without citrate addition [27]. They studied reduction potentials and sodium citrate complexes of these two elements. They reported that at $\mathrm{pH}$ values up to $4.5, \mathrm{H}_{3}$ Cit is abundantly present because of its stability, it does not make complexes with metal ions. When solution $\mathrm{pH}$ is increased, $\mathrm{H}_{2} \mathrm{Cit}^{-}$, $\mathrm{HCit}^{2-}$ and $\mathrm{Cit}^{3-}$ show peak values at around $4,5.7$, and 8 respectively. Beyond $\mathrm{pH}=8.5$ only $\mathrm{Cit}^{3-}$ is found to exist. They found that while CV of copper showed a reduction peak at $-0.1 \mathrm{~V}$ (vs. $\mathrm{Ag} / \mathrm{AgCl}$ ), when no citrate was added, this peak disappeared and a new peak formed at -0.8 when sodium citrate was added.

S.J. Kim et al. reported that in the $\mathrm{pH}=$ $5.8-6.5$ interval, depending on $\mathrm{Cu}$ concentration, $\left(\mathrm{Cu}_{2} \mathrm{Cit}_{2}(\mathrm{OH})_{2}\right)^{4-}$ is reported to be the most stable complex species in solution and it suppresses the direct reduction of $\mathrm{Cu}$ from the electrolyte [28].

Sangmin Shin et al. conducted a similar study for zinc as well. They reported that at $\mathrm{pH}=4.7, \mathrm{Zn}(\mathrm{Cit})_{2}^{4-}$ is the most stable complex species, but $\mathrm{H}_{2} \mathrm{Cit}^{2-}$ and $\mathrm{HCit}^{2-}$ ions coexist and the reduction of $\mathrm{Zn}$ is easier than at high $\mathrm{pH}$. Broad peaks at $-0.7 \mathrm{~V}$ were observed, which means that the reduction potential of $\mathrm{Zn}$ from citrate complex is increased. In brief, use of trisodium citrate makes codeposition of copper and zinc possible by bringing their reduction potentials close to each other.
Chemical composition of samples was revealed by MPAES method. Table I gives ultrasonication time vs film composition data. It is seen that chemical composition seems to be not so much affected by ultrasonication and there is no clear correlation between ultrasonication time and chemical composition of resultant samples. U15 and U60 have the lowest and the highest amount of copper with $35 \%$ and $48 \%$, respectively. All other samples have $\mathrm{Cu}$ compositions in $42-45 \%$ interval. Difference in chemical composition can again be explained by the type and/or amount of complexes in the solutions.

TABLE I

Chemical composition of thin films.

\begin{tabular}{c|c|c|c}
\hline \hline Sample & Zn $[\mathrm{ppm}]$ & $\mathrm{Cu}[\mathrm{ppm}]$ & $\mathrm{At. \% \textrm {Cu }}$ \\
\hline U0 & 4240 & 3380 & 44 \\
U5 & 3680 & 3010 & 45 \\
U10 & 6480 & 4950 & 43 \\
U15 & 6450 & 3490 & 35 \\
U20 & 4060 & 3120 & 43 \\
U25 & 6090 & 4980 & 45 \\
U30 & 9090 & 7440 & 45 \\
U45 & 9510 & 6870 & 42 \\
U60 & 7290 & 6660 & 48
\end{tabular}

\section{Conclusions}

CuZn alloy films were fabricated from aqueous citrate solutions. Sulphates of $\mathrm{Cu}$ and $\mathrm{Zn}$ were used as ion sources. TSC was be used as a complexing agent. It was aimed to change solution equilibrium conditions by applying high energy ultrasonication, which was considered as a tool to obtain films with different properties. CV results show that both anodic and cathodic current values are lower for non-ultrasonicated solution, compared to ultrasonicated ones. Furthermore shapes of CV curves show some differences between non-ultrasonicated reference sample in comparison to all of the ultrasonicated ones. CV graphs of ultrasonicated samples are similar to each other, regardless of ultrasonication period. Chemical compositions of samples are close to each other, and are around 44 at.\% $\mathrm{Cu}$, with extremities of $35 \%$ and $48 \%$ for 15 and 60 minutes ultrasonicated samples, respectively. Differences in CV graphs and chemical composition of samples may be attributed to type and/or amount of equilibrium complex species, formed in solutions, or some other changes which affect the mobility of charged species.

\section{References}

[1] F.A. Lowenheim, Modern Electroplating, Wiley, New York 1974.

[2] A. Brenner, Electrodeposition of Alloys. Principles and Practice, Vol. 1, Academic Press, New York 1963.

[3] H. Strow, Metal Finishing Guidebook 99, 206 (2001). 
[4] I.A. Carlos, M.R.H. de Almeida, J. Electroanal. Chem. 562, 153 (2004).

[5] F.B.A. Ferreira, F.L.G. Silva, A.S. Luna, D.C.B. Lago, L.F. Senna, J. Appl. Electrochem. 37, 473 (2007).

[6] R. Juskenas, V. Karpaviciene, V. Pakstas, A. Selskis, V. Kapocius, J. Electroanal. Chem. 602, 237 (2007).

[7] K.M. Ismail, R.M. Elsherif, W.A. Badawy, Electrochim. Acta 49, 5151 (2004).

[8] M. Hemmous, A. Layadi, A. Guittoum, L. Kerkache, N. Tiercelin, A. Klimov, V. Preobrazhensky, P. Pernod, Europ. Phys. J. Appl. Phys. 70, 10301 (2015).

[9] Kribalis, P.E. Tsakiridis, C. Dedeloudis, E.J. Hristoforou, J. Optoelectronics Adv. Mater. 8, 1475 (2006).

[10] A.S. Kadırbayeva, A.B. Bayeshov, Acta Phys. Pol. A 128, B-458 (2015).

[11] W. Laslouni, Z. Hamlati, M. Azzaz, Acta Phys. Pol. A 128, B-190 (2015).

[12] W. Laslouni, M. Azzaz, Acta Phys. Pol. A 130, 112 (2016).

[13] S.U. Rehman, M. Khan, A. Nusair Khan, L. Ali, S.H. Imran Jaffery, Acta Phys. Pol. A 128, B-125 (2015).

[14] X.B. Ren, K. Otsuka, MRS Bull. 27, 115 (2002).

[15] Yongqing Fu, Hejun Du, Weimin Huang, Sam Zhang, Min Hu, Sens. Actuators A: Phys. 112, 395 (2004).

[16] P. Krulevitch, A.P. Lee, P.B. Ramsey, J.C. Trevino, J. Hamilton, M.A. Northrup, J. MEMS 5, 270 (1996).
[17] E. Makino, M. Uenoyama, T. Shibata, Sens. Actuator A: Phys. 71, 187 (1998).

[18] I.N. Qureshi, M. Shahid, A.N. Khan, Acta Phys. Pol. A 128, B-314 (2015).

[19] N. Syla, F. Aliaj, B. Dalipi, Acta Phys. Pol. A 130, 83 (2016).

[20] S. Kırtay, Acta Phys. Pol. A 128, B-90 (2015).

[21] D. De Filippo, A. Rossi, D. Atzei, J. Appl. Electrochem. 22, 64 (1992).

[22] I.A. Carlos, M.R.H. de Almeida, J. Electroanal. Chem. 562, 153 (2004).

[23] P. Fricoteaux, C. Rousse, J. Electroanal. Chem. 733 , 53 (2014)

[24] N. Haberkorn, M. Ahlers, F.C. Lovey, Scripta Mater. 61, 821 (2009).

[25] I. Tudela, Y. Zhang, M. Pal, I. Kerr, T.J. Mason, A.J. Cobley, Surf. Coat. Technol. 264, 49 (2015).

[26] M. Kim, F. Sun, J. Lee, Y.K. Hyun, D. Lee, Surf. Coat. Technol. 205, 2362 (2010).

[27] Sangmin Shin, Chansu Park, Chiho Kim, Yangdo Kim, Sungkyun Park, Jae-Ho Lee, Curr. Appl. Phys. 16, 207 (2016).

[28] S.J. Kim, D.J. Duquette, J. Electrochem. Soc. 153 , C417 (2006). 